\title{
Ferromagnetic Resonance Study of Exchange Coupled (Ga,Mn)As/GaAs/(Ga,Mn)As Heterostructures
}

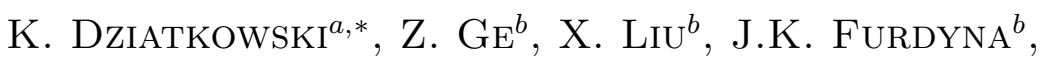 \\ B. ClerJaud ${ }^{c}$ AND A. Twardowski ${ }^{a}$ \\ ${ }^{a}$ Institute of Experimental Physics, Warsaw University \\ Hoża 69, 00-681 Warsaw, Poland \\ ${ }^{b}$ Department of Physics, University of Notre Dame \\ Notre Dame, Indiana 46556, USA \\ ${ }^{c}$ Institute des NanoSciences de Paris, 75015 Paris \\ and France Université Pierre et Marie Curie, 75005 Paris, France
}

Ferromagnetic resonance study of the exchange coupled (Ga,Mn)As/ GaAs/(Ga,Mn)As heterostructures is reported. The measurements were performed on the series of samples with varying thicknesses $d_{\mathrm{GaAs}}$ of nonmagnetic GaAs spacer, established $d_{\mathrm{GaAs}}$-dependent extent of weak and strong interlayer exchange coupling, judging on the observation of one or two ferromagnetic resonance modes.

PACS numbers: 75.50.Pp, 76.50.+g, 75.70.Cn, 75.30.Et, 85.75.-d

\section{Introduction}

Gallium arsenide with manganese is one of the most interesting representatives of diverse family of ferromagnetic semiconductors, and for several years has stayed under intense experimental and theoretical investigation. This huge scientific effort is triggered by the hope of using ( $\mathrm{Ga}, \mathrm{Mn}) \mathrm{As}$ as a material for the purposes of all-semiconductor spin electronics [1]. Among the fundamental spintronic structures are spin valve device (SVD) and magnetic tunnel junction (MTJ), both built of two ferromagnetic (FM) layers separated by a thin nonmagnetic (NM) film [2]. A magnitude of electric current passed throughout these structures is

${ }^{*}$ corresponding author; e-mail: konrad.dziatkowski@fuw.edu.pl 
modulated by the relative orientation of magnetization vectors of two ferromagnetic layers - reaching its maximum for parallel and minimum for antiparallel configuration - even though a physical mechanism behind the modulation is different for both devices: SVD operates on the basis of giant magnetoresistive effect (GMR), while MTJ employs tunnel magnetoresistance (TMR).

The experimental studies of FM/NM/FM trilayers based on ferromagnetic (Ga,Mn)As were until now limited only to magnetometric [3] and magnetotransport $[4,5]$ measurements, in the latter case revealing — for example - a magnitude of tunnel magnetoresistance reaching 290\% [6]. Independently numerous experiments were performed for (Ga,Mn)As/NM superlattices, and showed ferromagnetic intra- and interlayer ordering, provided the width of each ( $\mathrm{Ga}, \mathrm{Mn}) \mathrm{As}$ layer was larger than 5-7 nm [7-9]. This observation was confirmed to hold true even for zero-field-cooling measurements. Although some of these papers refer to anisotropic character of magnetism in ( $\mathrm{Ga}, \mathrm{Mn}) \mathrm{As}$, the comprehensive understanding of magnetic anisotropy in ( $\mathrm{Ga}, \mathrm{Mn})$ As-based SVD and MTJ devices is still lacking. Among many phenomena involved, one of the particular concern is dependence of magnetic anisotropy and interlayer exchange coupling (IEC) on nonmagnetic spacer thickness $d_{\mathrm{NM}}$. Establishing of $d_{\mathrm{NM}}$-dependent extent of weak and strong IEC is on the other hand crucial in view of independent control of ferromagnetic films in FM/NM/FM heterostructure. For these reasons we performed ferromagnetic resonance (FMR) study for a series of (Ga,Mn)As [9 $\mathrm{nm}] / \mathrm{GaAs} /$ $(\mathrm{Ga}, \mathrm{Mn}) \mathrm{As}[14 \mathrm{~nm}]$ trilayers with a thickness of nonmagnetic GaAs spacer varying in the range of $3-12 \mathrm{~nm}$.

\section{Experimental}

The samples in study were grown in a Riber $32 \mathrm{R} \& \mathrm{D}$ molecular beam epitaxy (MBE) system. On semi-insulating GaAs(001) substrates a sequence of high$(135 \mathrm{~nm})$ and low-temperature $(2 \mathrm{~nm})$ GaAs buffers was deposited, followed by a heterostructure consisting of two ferromagnetic (Ga,Mn)As films (9 and $14 \mathrm{~nm}$ ) separated by a nonmagnetic GaAs spacer. The width of the latter layer was varied from sample to sample. In order to obtain a significant amount of Mn atoms in $(\mathrm{Ga}, \mathrm{Mn}) \mathrm{As}$ alloy, a low temperature MBE growth was performed at substrate temperature of about $250^{\circ} \mathrm{C}$. An intentional composition of manganese was $5 \%$, however, a further structural study revealed some fluctuations of magnetic ions content in the range of $3.8-5.6 \%$. For the whole series a growth process was monitored in situ by a reflection high energy electron diffraction (RHEED) device, which revealed a $1 \times 2$ surface reconstruction pattern confirming a good crystalline quality of deposited materials.

FMR experiments were performed with the use of a Varian electron spin resonance (ESR) spectrometer working at X-band microwave frequency $(\approx 9.3 \mathrm{GHz})$ and equipped with a continuous-flow helium cryostat. All measurements were carried out at the lowest available temperature of about $3 \mathrm{~K}$, with microwave ir- 
radiation power set up to $25 \mathrm{~mW}$. Each sample was fastened to quartz-rod holder subsequently in three different configurations, with the purpose of collecting FMR spectra for external magnetic field $\boldsymbol{H}$ aligned to four principal crystallographic axes: [100], [110], [1]10], and [001].

\section{Results and discussion}

The FMR spectrum shown in Fig. 1a was collected for the sample with $3 \mathrm{~nm}$ thin GaAs spacer and with magnetic field $\boldsymbol{H}$ along [110] crystallographic direction. Despite two structurally separated ferromagnetic films in the studied heterostructure, the only one FMR resonance line was observed for any orientation of $\boldsymbol{H}$ in the sample plane, not only for main crystallographic axes. This observation clearly demonstrates the IEC being so strong that the whole sample behaves as a single ferromagnetic film with some effective magnetic parameters. It is worth noting that according to Heinrich and Cochran [10] these effective parameters (e.g. magnetic anisotropy constants) are the magnetization-weighted mean values of the quantities corresponding to component ferromagnetic films. For heterostructures with thicker GaAs spacer, one or two resonance lines were observed in FMR spectra recorded for $\boldsymbol{H}$ kept in the sample plane. Figure 1b presents a typical example of such 2-line spectrum. In each of them one of two lines is much stronger than another one, but the ordering of this pair depends on the orientation of magnetic field with respect to crystallographic directions which will be addressed below.

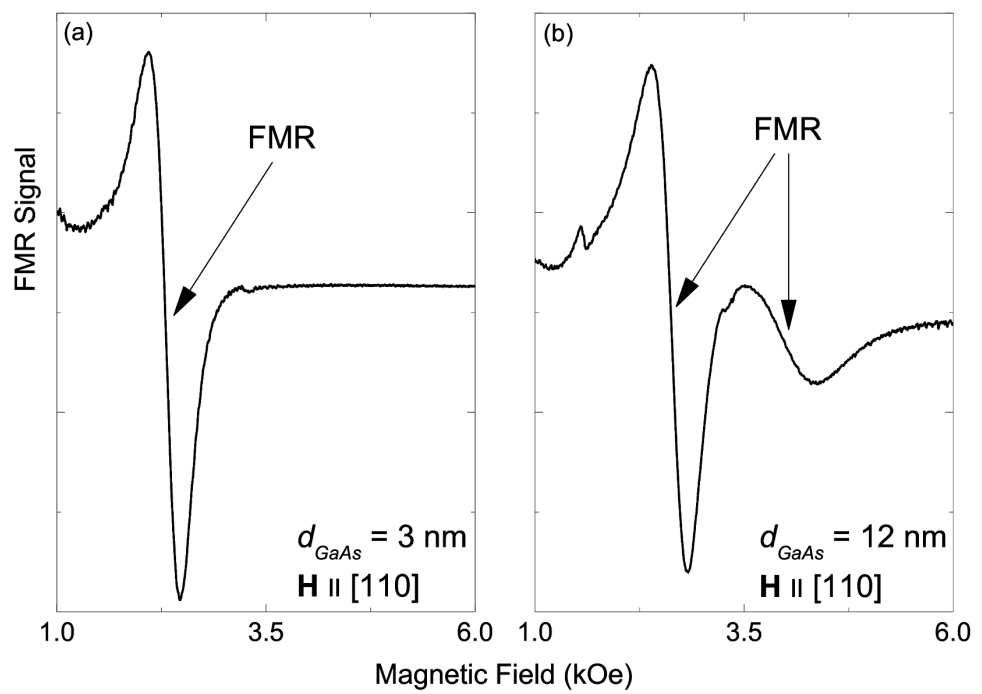

Fig. 1. Typical FMR spectra of (Ga,Mn)As/GaAs/(Ga,Mn)As heterostructures with (a) $3 \mathrm{~nm}$ and (b) $12 \mathrm{~nm}$ thick GaAs spacer. For the latter case two resonance lines are present, whereas the small peak at about $1.5 \mathrm{kOe}$ originates from contamination. The external magnetic field was applied along [110] crystallographic axis. 
A similar observation of 2-line spectra holds for magnetic field $\boldsymbol{H}$ oriented along growth axis [001], again with different magnitudes of the recorded FMR resonance lines.

The resonance fields for main crystallographic axes are collected in Fig. 2, where qualitative description of line magnitudes is also depicted. One may easily notice that both in-plane and out-of-plane data confirm the dependence of IEC on the spacer thickness $d_{\mathrm{GaAs}}$, and for any direction of magnetic field the line splitting advances with rising $d_{\mathrm{GaAs}}$. As expected, a wider structural separation of two ferromagnetic films results in weaker IEC. It was mentioned above that for $d_{\mathrm{GaAs}} \leq$ $3 \mathrm{~nm}$ IEC is strong enough to keep magnetization vectors of both ferromagnetic films fully coupled in (001) sample plane, thus only one resonance line is observed. With larger thickness of GaAs spacer two FM films decouple gradually, which is evidenced by rising line splitting for both in-plane $(\boldsymbol{H} \|[100],[110],[1 \overline{1} 0])$ and out-of-plane $\left(\boldsymbol{H} \|\right.$ [001]) configurations. Eventually for $d_{\mathrm{GaAs}} \geq 9 \mathrm{~nm}$ the line splitting seems to saturate and remains roughly constant, suggesting that $9 \mathrm{~nm}$ thick GaAs spacer separates (Ga,Mn)As layers sufficiently in view of independent or almost independent behavior of ferromagnetic films.
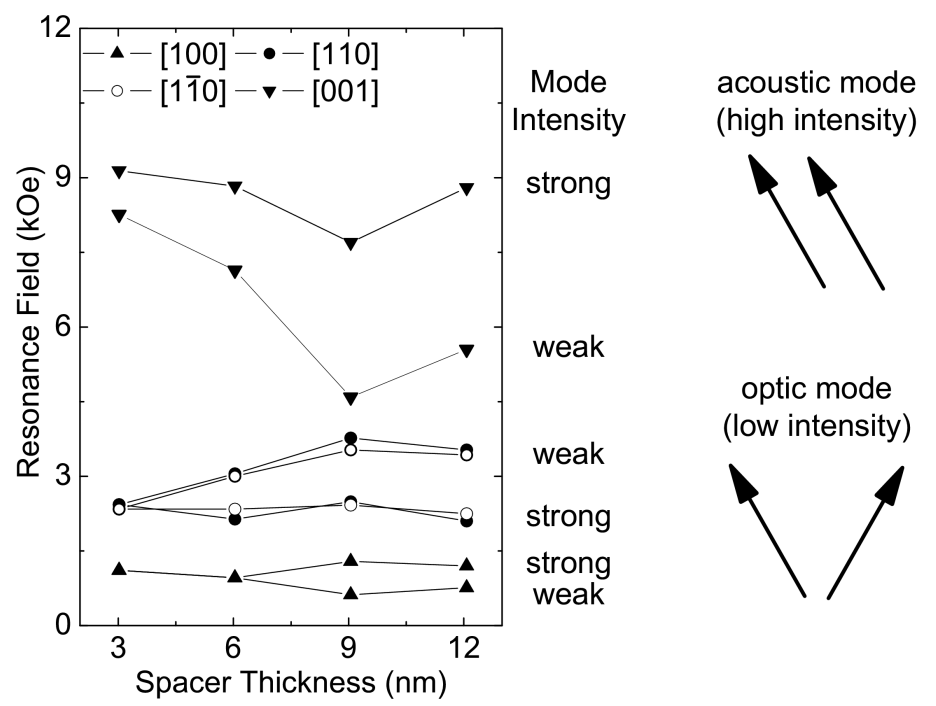
(low intensity)

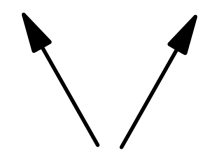

Fig. 2. Resonance magnetic fields (for main crystallographic directions) plotted versus thickness of nonmagnetic GaAs spacer. The line splitting clearly advances with rising $d_{\mathrm{GaAs}}$, indicating simultaneous decrease in IEC. On the right-hand side the magnitudes of observed modes are qualitatively depicted, with their supposed identification based on $[10]$.

Finally the origin of two lines in collected FMR spectra needs to be addressed. It is customary to attribute these lines with different excitations of an entire exchange-coupled FM/NM/FM heterostructure [10]. Within classical de- 
scription of ferromagnetic resonance, one line corresponds to the situation when magnetic moments of both FM films precess in phase (it is so-called acoustic mode, see Fig. 2), while another line appears for antiphase precession (an optic mode). In the latter case, a non-zero exchange interaction between magnetic moments of two FM films results in the additional torque exerted on one FM film by another one. This torque - via the Landau-Lifshitz equation of motion - makes the resonance field of the optic mode to be different from that observed for the acoustic mode, in which case no additional torque is present. The microwave radiation couples to a transversal (with respect to $\boldsymbol{H}$ ) component of the magnetic moment and the intensity of each mode is proportional to net magnitude of moment's transversal constituent. Thus acoustic mode reveals a higher intensity than its optic counterpart. In particular, an optic mode cannot be observed in symmetric FM/NM/FM trilayers, when the transversal component of magnetic moment vanishes. An appearance of two resonance lines in the spectra presented in this paper suggests that the above-mentioned explanation may be valid also in our study. In such case, however, the ordering of these two lines in FMR spectrum depends on a type of interlayer exchange coupling: for ferromagnetic IEC the large-intensity mode appears at higher magnetic field than the weaker one; for antiferromagnetic IEC the order is reversed [10]. As shown in Fig. 2 the ordering of two lines in our FMR spectra changes with respect to the orientation of external magnetic field $\boldsymbol{H}$. But it is rather unlikely that the type of IEC in $(\mathrm{Ga}, \mathrm{Mn}) \mathrm{As} / \mathrm{GaAs} /(\mathrm{Ga}, \mathrm{Mn})$ As heterostructure changes from ferromagnetic to antiferromagnetic with rotation of $\boldsymbol{H}$ (e.g. from [100] to [110]). At the moment we are not in position to provide a conclusive explanation of this phenomenon, which readily needs further studies and reaches beyond the capacity of our short communication.

\section{Conclusions}

In summary, we presented ferromagnetic resonance study of the exchange coupled $(\mathrm{Ga}, \mathrm{Mn}) \mathrm{As} / \mathrm{GaAs} /(\mathrm{Ga}, \mathrm{Mn})$ As trilayers with different thicknesses of nonmagnetic GaAs film. It was shown that for $d_{\mathrm{GaAs}} \leq 3 \mathrm{~nm}$ the heterostructure was fully coupled and behaved as a single (Ga,Mn)As layer, provided that external magnetic field $\boldsymbol{H}$ was kept in-plane. It was evidenced by the observation of a single FMR line for $\boldsymbol{H} \perp$ [001]. With rising $d_{\mathrm{GaAs}}$ the IEC decreases as two resonance lines were detected in FMR spectra. For $d_{\mathrm{GaAs}} \geq 9 \mathrm{~nm}$ the line splitting saturates and two component ferromagnetic films seem to be decoupled.

\section{References}

[1] T. Dietl, Nature Mat. 2, 646 (2003).

[2] S.A. Wolf, D.D. Awschalom, R.A. Buhrman, J.M. Daughton, S. von Molnár, M.L. Roukes, A.Y. Chtchelkanova, D.M. Treger, Science 294, 1488 (2001).

[3] N. Akiba, F. Matsukura, A. Shen, Y. Ohno, H. Ohno, A. Oiwa, S. Katsumoto, Y. Iye, Appl. Phys. Lett. 73, 2122 (1998). 
[4] T. Hayashi, H. Shimada, H. Shimizu, M. Tanaka, J. Cryst. Growth 201/202, 689 (1999).

[5] D. Chiba, N. Akiba, F. Matsukura, Y. Ohno, H. Ohno, Appl. Phys. Lett. 77, 1873 (2000).

[6] D. Chiba, F. Matsukura, H. Ohno, Physica E 21, 966 (2004).

[7] T. Hayashi, M. Tanaka, K. Seto, T. Nishinaga, K. Ando, Appl. Phys. Lett. 71, 1825 (1997).

[8] A. Shen, H. Ohno, F. Matsukura, H.C. Liu, N. Akiba, Y. Sugawara, T. Kuroiwa, Y. Ohno, Physica B 249-251, 809 (1998).

[9] H. Kepa, J. Kutner-Pielaszek, A. Twardowski, C.F. Majkrzak, J. Sadowski, T. Story, T.M. Giebultowicz, Phys. Rev. B 64, 121302(R) (2001).

[10] B. Heinrich, J.F. Cochran, Adv. Phys. 42, 523 (1993). 